\title{
Evaluation of a new stent for EUS-guided pancreatic duct drainage: long-term follow-up outcome
}

다 $(1) \div$

\author{
Authors \\ Yukitoshi Matsunami ${ }^{1}$, Takao Itoi ${ }^{1}$, Atsushi Sofuni ${ }^{1}$, Takayoshi Tsuchiya ${ }^{1}$, Kentaro Kamada ${ }^{1}$, Reina Tanaka ${ }^{1}$, Ryosuke

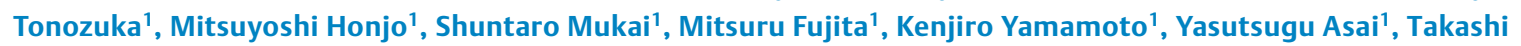 \\ Kurosawa', Shingo Tachibana ${ }^{2}$, Yuichi Nagakawa²
}

Institutions

1 Department of Gastroenterology and Hepatology, Tokyo Medical University, Tokyo, Japan

2 Department of Gastrointestinal and Pediatric Surgery, Tokyo Medical University, Tokyo, Japan

submitted 8.6.2017

accepted after revision 1.12 .2017

\author{
Bibliography \\ DOI https://doi.org/10.1055/s-0044-101753 | \\ Endoscopy International Open 2018; 06: E505-E512 \\ (c) Georg Thieme Verlag KG Stuttgart · New York \\ ISSN 2364-3722
}

Corresponding author

Takao Itoi, MD, PhD, FASGE, Department of

Gastroenterology and Hepatology, Tokyo Medical

University, 6-7-1 Nishishinjuku, Shinjuku-ku, Tokyo

160-0023, Japan

Fax: +81-3-5381-6654

itoi@tokyo-med.ac.jp

\section{ABSTRACT}

Background and study aims Endoscopic ultrasonography-guided pancreatic duct drainage (EUS-PD) has been reported as an alternative for failed conventional endoscopic retrograde cholangiopancreatography (ERCP). However, there are few dedicated devices for EUS-PD. Recently, we have developed a new plastic stent dedicated to EUS-PD and have conducted a feasibility study to evaluate its efficacy. In the current study, we evaluated the long-term efficacy of this new plastic stent.

Patients and methods Thirty patients $(61 \pm 14.3$ years old, 14 men) with acute recurrent pancreatitis caused by a stricture in the main pancreatic duct (MPD) or stenotic pancreatoenterostomy were treated at our institution using our recently developed 7Fr plastic stent between August 2013 and April 2017.

Results The stent was placed successfully in all patients (30/30) and early clinical success was achieved in all of them. Early adverse events (AEs) occurred in seven patients (23.3\%), namely, self-limited abdominal pain $(n=5)$, mild pancreatitis $(n=1)$, and bleeding which required transcatheter arterial embolization $(n=1)$. Two patients died of primary disease and three were lost to follow-up. The remaining 25 patients were followed up after initial EUS-PD for a median of 23 months (range, 6-44 months). Twenty patients required regular stent exchange ( 3 times; range, 1 12 times). Spontaneous stent dislodgement was observed in six patients. Four patients wanted their stents removed 1 year after the initial intervention. Twelve patients (48\%) had regular stent exchange 1 year after the initial intervention. Three patients converted to standard transpapillary pancreatic duct stenting by conventional ERCP. Finally, nine patients (36\%) had complete stent removal either intentionally or by spontaneous dislodgement without any symptoms.

Conclusion The new plastic stent for EUS-PD was associated with not only short-term technical success but also longterm clinical success in the majority of patients evaluated in this study.

\section{Introduction}

Acute recurrent pancreatitis (ARP) reduces quality of life for patients because of pain and limited food intake. ARP is usually observed in patients with a disconnected pancreatic duct (PD) or pancreatojejunostomotic stricture with or without a pancreatic fistula. Traditionally, ARP caused by PD disruption and obstruction has been treated by endoscopic retrograde cholan- giopancreatography (ERCP) in patients whose anatomy is unaltered, and more recently, balloon enteroscopy-assisted ERCP has been used in patients with Whipple resection.

Although ERCP remains the first-line therapy because it is a minimally invasive method, it may not always be successful. Thus, EUS-PD has recently been established [1], although it appears to be one of the most difficult interventional endoscopy techniques. Recent reports have revealed that the technical 
success rate of EUS-PD is in the range of $40 \%$ to $100 \%$ [2-8]. In fact, AEs such as pancreatic juice leakage, stent migration, and pancreatitis have occasionally been observed. One possible reason for the low technical success rate is the lack of a dedicated stent for EUS-PD. We have recently developed a new stent dedicated to EUS-PD and have reported preliminary outcomes with it in a feasibility study [2]. In that study, with a mean follow-up time of $7.4 \pm 4.1$ months, all eight patients were successfully treated with the new stent, resulting in a high clinical success rate of $100 \%(8 / 8)$ with only self-limited abdominal pain as a mild AE occurring in one patient. However, to the best of our knowledge, few clinical studies have described late AEs and long-term follow-up on outcomes with EUS-PD. Furthermore, the optimal time for stent removal remains controversial. Herein, we describe the long-term follow-up outcome of EUS-PD using our recently developed dedicated PD stent.

\section{Patients and methods}

\section{Patients}

The study included 30 consecutive patients (mean age, $61 \pm$ 14.3 years; 14 men) with ARP caused by a stricture of the MPD or stenotic pancreatojejunostomy who underwent EUS-PD stenting procedures from August 2013 to April 2017 at Tokyo Medical University Hospital ( $\triangleright$ Table 1 ). Clinical symptoms were defined as ARP symptoms, such as epigastric pain, as well as pain with hyperamylasemia and enlarged pancreatic duct with intraductal hypertension, which were observed within 6 months before intervention. Intervention was administered to ARP patients who had at least two or more attacks of pancreatitis within the 6 months before the intervention. Technical success was defined as stent placement in the MPD. Clinical success was defined as complete resolution of clinical symptoms, which included relief from both ARP symptoms and pain with hyperamylasemia and enlarged pancreatic duct. Early clinical success was defined as no recurrence of ARP symptoms after the first intervention and at 6-month follow-up (i.e., shortterm outcome). Late clinical success was defined as no recurrence of ARP symptoms during the follow-up time during which detailed follow-up data on outcomes were available (i. e., longterm outcome). AEs were classified into three types: acute AEs, early AEs ( $\leq 1$ month of the procedure), and late AEs (> 1 month after the procedure). AEs were graded according to the severity grading system of the American Society for Gastrointestinal Endoscopy Lexicon [9]. We defined "stent migration" as migration of the stent into the pancreatic duct, that is, inward migration. That could result in severe AEs as retrieving a stent that has migrated into the pancreatic duct is technically challenging. We defined "stent dislodgement" as stent migration into the stomach side, that is, outward migration. In contrast, that does not cause severe AEs in majority of patients as a stent can be removed by endoscopy or eliminated with stool.

\section{EUS-PD procedure}

All EUS-PD procedures were performed after hospital admission. Patients were hospitalized for 3 days before the procedure, and food and drinks were not allowed 1 day before the
- Table 1 Patient characteristics $(n=30)$.

Mean age (years) $\pm S D$

$60.6 \pm 14.3$

Sex

- Male/Female

$14 / 16$

Indication

- Acute recurrent pancreatitis

30

Prior ERP attempted

$19(66 \%)$

Underlying diseases

- Stenotic pancreaticojejunostomy (DPDS)

$19(1)$

- Chronic pancreatitis (DPDS)

- Stenotic pancreaticogastrostomy

2

- Pancreatic divisum 1

- Pancreatic cancer (DPDS)

Surgically altered anatomy

- PD 5

- PpPD

10

- SSPPD 3

- PD + pancreaticogastrostomy

- Middle pancreatectomy

- None 9

Stricture site

- Pancreatoenteric anastomosis 21

- Pancreatic duct 9

ERP, endoscopic retrograde pancreatography; DPDS, disconnected pancreatic duct syndrome; PD, pancreaticoduodenectomy; PpPD, pylorus-preserving pancreaticoduodenectomy; SSPPD, subtotal stomach preserving pancreaticoduodenectomy

procedure. Serum amylase level was checked in all patients 1 day before the procedure and 1 day and 1 month after the procedure. A therapeutic curved linear array echoendoscope was used under carbon dioxide insufflation. After confirming no intervening vessels using the Doppler mode, the pancreatic duct was punctured transgastrically using a 19G or 22G fine-needle aspiration needle under EUS guidance. We punctured a more distal part of the PD as far from the papilla or anastomotic site as possible $(>3 \mathrm{~cm})$ because the PD length is sufficient for placing the stent tip even in case of inability to advance the guidewire across the papilla and anastomotic site. After performing pancreatography, a 0.025-inch guidewire (VisiGlide 2, Olympus Medical Systems, Tokyo, Japan) was inserted into the main pancreatic duct and advanced across the papilla or anastomotic site if possible. When a $22 \mathrm{G}$ needle was used, a thinner guidewire (0.021-inch Metro, COOK Medical, Tokyo, Japan, or 0.018inch Pathfinder, Boston Scientific Japan, Tokyo, Japan) was used. There are some reports in the literature of peeling of the guidewire in case of interventional EUS $[10,11]$. To prevent such AEs, gentle guidewire manipulation is necessary, particu- 
larly when using a thinner wire, because the guidewire may kink at the tip. When guidewire manipulation is difficult under the needle, after needle tract dilation, the needle is removed and the standard injection catheter (ERCP catheter, MTW Co., Dusseldorf, Germany) is advanced into the pancreatic duct over the wire. This helps provide traction for wire manipulation. We used a 0.025 -inch VisiGlide2 guidewire as it has sufficient stiffness at the wire shaft and kinks less than a thinner guidewire. An electrocautery dilator (6.5 Fr, Cyst-Gastro set, Endoflex, Voerde, Germany), a mechanical dilator, or a 4-mm-diameter dilating balloon (Hurricane, Boston Scientific, Natick, Massachusetts, United States) was used as bougie for the needle tract. Before March 2016, our first choice for dilation of the needle tract was an electrocautery dilator, which takes less time for dilation than other dilators and prevents pancreatic fibrosis. However, diathermy catheters cause an acute and late burn effect around the needle tract, leading to serious AEs including pancreatitis and bleeding. For these reasons, in April 2016, we started using a mechanical dilator for tract dilation. That dilator is plastic and has a tip that is extremely tapered up to $2.5 \mathrm{Fr}$. The tip is very fine and allows easy penetration, enables smooth insertion, and is less likely to cause bleeding and pancreatitis than an electrocautery dilator. Also, a 4-mm-diameter dilating balloon was added to dilate the needle tract and stricture site according to the condition.

We have recently developed a new 7Fr single pigtail type plastic stent, which has a total length of $20 \mathrm{~cm}$ and an effective length of $15 \mathrm{~cm}$ for all patients [2]. That stent has a pigtail anchor in the proximal end and four internal flanges ( 2 distal and 2 proximal) to prevent stent migration. There are relatively large apertures below the flanges and four small side holes (2 distal and 2 proximal). Absence of holes in the middle part of the stent can prevent pancreatic juice leakage. Moreover, this new stent has a straight and tapered tip and it has improved ability to traverse strictures compared with a stent with a distal pigtail and is also easier to push and insert than standard PD stents that are not tapered to the same extent. In patients with passage in the anastomotic site or papilla, the stent was placed over the papilla or anastomotic site for drainage in both directions (i.e., transenteric antegrade PD stenting). In patients without passage in the anastomotic site or papilla, the distal part of the stent was placed in the MPD and the proximal end remained in the stomach (i.e., pancreaticoenterostomy) (CX-T stent, TYPE IT, Gadelius Medical Co., Tokyo, Japan) (ฉ Fig. 1 and $>$ Fig. 2).

All patients in whom EUS-PD was performed underwent abdominal ultrasound or computed tomography (CT) 1 month after the first intervention for postinterventional imaging to check for a smaller PD diameter.

\section{Follow-up strategy}

Stent exchange was planned every 3 to 4 months during the year after initial stent placement unless spontaneous dislodgement occurred. Patients who did not want to retain the stent had their stent removed completely. In patients with surgically altered anatomy who opted for regular stent exchange, the stent was continuously exchanged every 3 to 4 months. In pa-

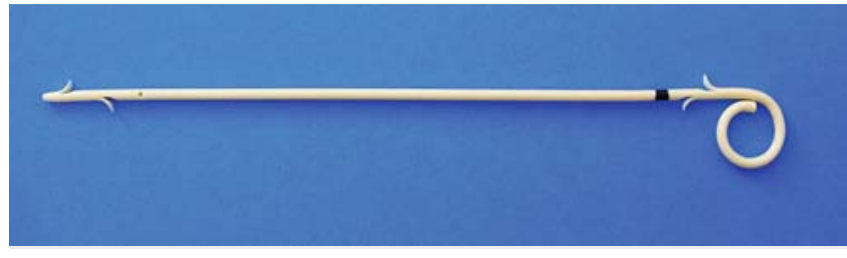

Fig. 1 EUS-PD dedicated plastic stent.

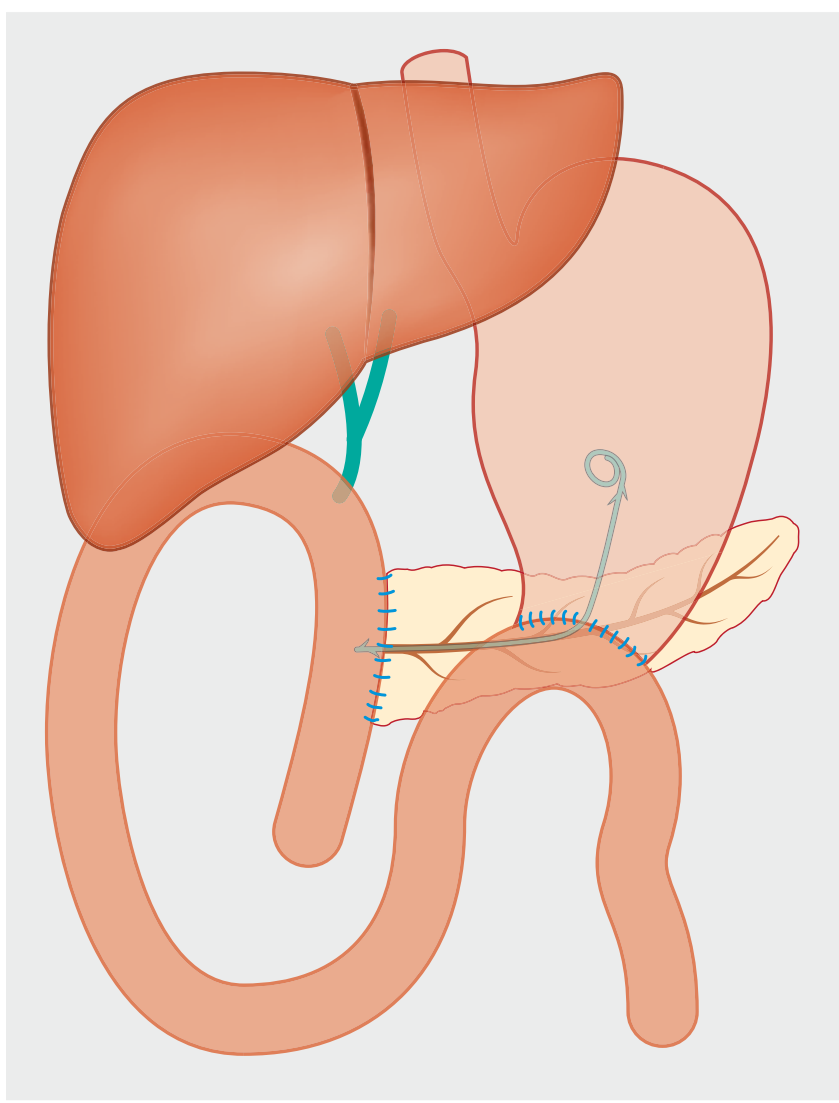

- Fig. 2 Schema of EUS-guided PD stenting in postsurgical anatomy patients.

tients with normal anatomy, at the time of stent exchange, if the guidewire advanced across the stricture site and papilla into the duodenum, the EUS-PD stent was removed and the usual pancreatic stent was deployed via the papilla. Subsequently, the pancreatic stent was continuously exchanged every 3 to 4 months. One year later, if a patient did not want to retain the stent, the stent was removed completely. If a patient opted for regular stent exchange, the stent was continuously exchanged every 3 to 4 months.

The study protocol was approved by the institutional review board of Tokyo Medical University (No.2017041). All patients gave their written informed consent before use of the novel PD stent. 


\section{Statistical analysis}

Distribution of continuous variables pertaining to baseline characteristics of the two treatment groups in the cohorts were compared using the Student's t-test, Welch's t-test, or the Wilcoxon rank-sum test, as appropriate. Proportions of categorical variables were compared using the chi-squared or Fisher's exact test. All reported $P$ values are two-sided, and $P$ values $<0.05$ were considered to indicate a statistically significant difference. Analyses were performed using SPSS (version 20; SPSS, Chicago, Illinois, United States) or R software version 2.12.0.

\section{Results}

Patient characteristics, indications, underlying diseases, and reconstruction methods after pancreatoduodenectomy are summarized in - Table 1. The EUS-PD indication for all 30 patients was ARP. ERP was previously performed in 19 patients, including 10 patients with surgically altered anatomy and nine patients with normal anatomy. In all nine patients with normal anatomy, conventional ERP drainage was first attempted in our institution. However, passage into the papilla or stricture site by conventional ERP was not possible in those nine patients. In 21 patients with surgically altered anatomy, balloon enteroscopyassisted ERP was first attempted in 10 patients. However, that failed because the pancreaticoenteric anastomotic site was inaccessible. EUS-PD drainage was first attempted in 11 of the 21 patients in our institution because of the difficulty of surgical reconstruction or failed balloon enteroscopy-assisted ERP in a previous hospital. Disconnected pancreatic duct syndrome (DPDS) was observed in five patients who underwent pancreatojejunostomy $(n=1)$, with chronic pancreatitis $(n=2)$, and with pancreatic cancer $(n=2)$. All five DPDS patients underwent pancreaticogastrostomy because the guidewire did not pass through the disrupted site.

\section{Short-term outcome of EUS-PD}

Both the technical and clinical success rates of EUS-PD were $100 \%$ (30/30) ( Table 2). The median maximum PD diameter measured was $5.2 \mathrm{~mm}$ (range, $1-14 \mathrm{~mm}$ ), but the actual punctured PD diameter was significantly smaller than the maximum PD diameter (median, $3.5 \mathrm{~mm}$; range, $1-14 \mathrm{~mm}, P=0.014$ ). A $22 \mathrm{G}$ needle was used in 14 patients (46.7\%). The mean procedure time was $30.8 \pm 13.5$ minutes. Eventually, the stent could be placed across the pancreaticoenteric stricture site in 12 cases by transenteric antegrade PD stenting. However, in the remaining 18 patients, the guidewire could not be passed across the stricture and the stent tip was placed in the PD near the pancreaticogastrostomy anastomotic site.

There were no acute AEs or cases of peeling of the guidewire tip. Early AEs occurred in seven patients (23\%) (mild 5, moderate 1 , and severe 1 ). Self-limited abdominal pain was observed in five patients. Bleeding that required transcatheter arterial embolization was observed in one patient in whom an electrocautery dilator was used for tract dilation. The bleeding site was not observed during the procedure. However, 1 day after the

\begin{tabular}{|l|l|}
\hline \begin{tabular}{|l|}
\hline Table 2 Short-term outcome of EUS-PD $(\mathrm{n}=30)$ \\
\hline Technical success
\end{tabular} \\
\hline Clinical success & $30 / 30(100 \%)$ \\
\hline Early adverse events & $30 / 30(100 \%)$ \\
\hline - Mild pain & $7(23 \%)$ \\
\hline - Moderate pancreatitis & 5 \\
\hline - Severe bleeding & 1 \\
\hline Median maximum PD diameter $(\mathrm{mm})$ & 1 \\
\hline - Range (mm) & $5.2 \pm 3.3$ \\
\hline Median punctured PD diameter $(\mathrm{mm})$ & $1-14$ \\
\hline - Range (mm) & $3.5 \pm 2.9$ \\
\hline Mean procedure time (min) & $1-14$ \\
\hline - Range (mm) & $30.8 \pm 13.5$ \\
\hline Needle used & $10-70$ \\
\hline - 19-gauge & 25 \\
\hline - 22-gauge & 17 \\
\hline Devices for tract dilation & 13 \\
\hline - Electrocautery dilator & \\
\hline - 4-mm-diameter dilating balloon & \\
\hline - Mechanical dilator & \\
\hline
\end{tabular}

procedure, CT showed a hematoma between the stomach and the pancreas. Extravasation and pseudoaneurysm were observed in the left gastric artery on transarterial embolization, and were treated by coil embolization. Pancreatitis was observed in one patient in whom an electrocautery dilator was used. However, the pancreatitis was moderate and treated by conservative therapy, although this caused a delay in discharge by 11 days. Hyperamylasemia was observed in six patients without any additional treatment.

\section{Long-term outcome of EUS-PD}

A flow chart of the long-term clinical outcome of EUS-PD is shown in Fig. 3. Detailed follow-up was available for 25 patients $(83.3 \%)$. Two patients died of primary malignant disorder and three were lost to follow-up and censored at the last date of examination. The rest of the 25 patients' median follow-up time after the initial EUS-PD was 23 months (range, 6-44 months). Among them, 20 patients underwent regular stent exchange (median, 3 times; range, $1-12$ times) ( $\triangleright$ Table 3 ).

During the median follow-up time of 23 months in the 25 patients, spontaneous stent dislodgement into the stomach occurred in six patients (24\%). Four patients whose ARP symptoms were relieved wanted their stents removed 1 year after the initial intervention because they did not want to continue with regular stent exchange. Twelve patients (48\%) have opted to undertake regular stent exchange even more than 1 year 


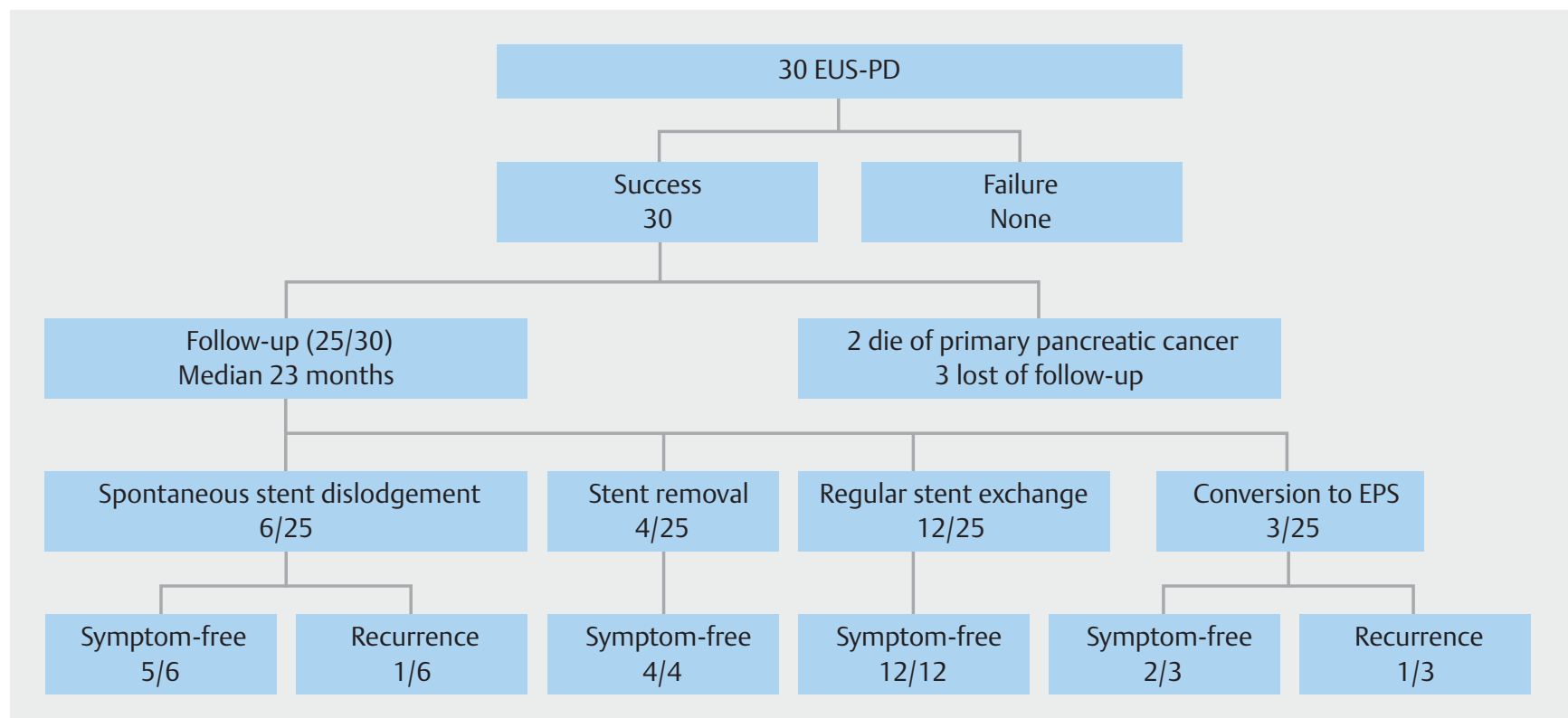

- Fig. 3 Flow chart of clinical outcome.

\begin{tabular}{|c|c|}
\hline Median follow-up after initial EUS-PD (months) & 23 \\
\hline - Range (months) & $6-44$ \\
\hline $\begin{array}{l}\text { Median number of procedures for stent exchange } \\
(n=20) \text { (times) }\end{array}$ & 3 \\
\hline - Range (times) & $1-12$ \\
\hline \multicolumn{2}{|l|}{ Late adverse events } \\
\hline - Stent dislodgement & $6 / 25(24 \%)$ \\
\hline Long-term clinical success rate & $23 / 25(92 \%)$ \\
\hline Recurrent pancreatitis & $2 / 25(8 \%)$ \\
\hline
\end{tabular}

after the first intervention ( $\triangleright$ Table 4 ). Three patients with normal anatomy converted to standard transpapillary PD stenting by conventional ERCP. Of the 25 patients, 10 patients had stent removal either intentionally or by spontaneous dislodgement, however, one of them had a recurrence of symptoms. Finally, nine patients (36\%) were completely symptom-free with stent removal. Long-term clinical success was achieved in 23 of the 25 patients $(92 \%)$ in whom long-term follow up was possible and recurrence occurred in two cases. One patient was a case of stenotic pancreaticojejunostomy after subtotal stomachpreserving pancreaticoduodenectomy (SSPPD). The stent was placed as a pancreaticoenterostomy. However, it spontaneously dislodged 6 months after the first intervention and MPD dilation was not improved. The patient was treated by reintervention of EUS-PD and the same stent was deployed. The other patient had alcoholic chronic pancreatitis with normal anatomy. Symptom recurrence occurred in that individual 12 months after the first intervention and 3 months after conversion to normal EPS. That patient was treated by stent exchange with conventional ERP. After that, there was no recurrence of symptoms in either patient. Long-term follow-up outcomes were clarified in three patients with DPDS. The underlying diseases were chronic pancreatitis in two patients and stenotic pancreaticojejunostomy in one patient. The pancreatic fistula was closed in two patients and their stent was removed in 1 year. One patient in whom the pancreatic fistula could not be closed underwent regular stent exchange more than 1 year after stent placement. However, surgical repair was not required in any case.

\section{Discussion}

The first case of EUS-guided pancreaticogastrostomy was reported by Francois et al [12] in 2002. Since then, several investigators have addressed the feasibility of EUS-PD [2-8]. Nonetheless, EUS-PD is still thought to be one of the most challenging procedures in interventional EUS for the following reasons: 1) a targeted PD is smaller than pancreatic fluid collections, the gallbladder, or even a dilated bile duct; 2) an echoendoscope is unstable in the large cavity of the stomach during EUS-PD; 3) the stomach is anatomically not adhered to the pancreas and a breathing change causes difficulty in needle puncture of the targeted PD compared with other interventional EUS procedures; and 4) there is currently no dedicated PD stent for EUSPD. In particular, a PD stent is the most important device because the procedure fails even after successful tract dilation without the PD stent, leading to serious AEs such as pancreatic juice leakage. Recently, we have developed a new dedicated EUS-PD stent with both technical and clinical success rates of $100 \%$ as shown in our previous feasibility study [2]. However, the ideal length of the EUS-PD stent is unclear [13]. The effective length of this new stent is $15 \mathrm{~cm}$. For patients in whom pas- 
- Table 4 Patients who have undertaken regular stent exchange after 1 year $(n=12)$.

\begin{tabular}{|l|l|l|l|l|}
\hline Patient \# & Age, years/sex & Underlying disease & $\begin{array}{c}\text { Number of stent } \\
\text { exchanges }\end{array}$ & $\begin{array}{l}\text { Total follow-up } \\
\text { time }\end{array}$ \\
\hline 1 & $57 /$ female & Chronic pancreatitis & 2 & 13 months \\
\hline 2 & $44 /$ male & Stenotic pancreaticojejunostomy & 2 & 23 months \\
\hline 3 & $52 /$ male & Chronic pancreatitis & 3 & 12 months \\
\hline 4 & $26 /$ female & Stenotic pancreaticojejunostomy & 3 & 16 months \\
\hline 5 & $53 /$ female & Stenotic pancreaticojejunostomy & 3 & 16 months \\
\hline 6 & $59 /$ male & Chronic pancreatitis & 5 & 16 months \\
\hline 7 & $68 /$ male & Stenotic pancreaticojejunostomy & 5 & 22 months \\
\hline 8 & $52 /$ female & Stenotic pancreaticojejunostomy & 5 & 25 months \\
\hline 9 & $65 /$ male & Stenotic pancreaticojejunostomy & 6 & 27 months \\
\hline 10 & $62 /$ male & Stenotic pancreaticojejunostomy & 6 & 32 months \\
\hline 11 & $35 /$ female & Stenotic pancreaticojejunostomy & 9 & No \\
\hline 12 & $79 /$ female & Stenotic pancreaticojejunostomy & 12 & 36 months \\
\hline
\end{tabular}

sage into the anastomosis site or papilla was achieved, the stent was placed across the anastomosis site or papilla, and the stent length of $15 \mathrm{~cm}$ was a suitable size for drainage in both directions. Rendezvous ERP stent placement is one option in case of guidewire passage into the anastomosis site or papilla. Previously we performed rendezvous ERP stent placement. However, it took time to change the scope and the procedure was more complicated than EUS-PD, especially in patients whose anatomy was surgically altered. There was also concern about pancreatic juice leakage with a long procedure for the first intervention. Compared to the rendezvous technique, EUS-PD takes less time and is much simpler. Also, regular stent exchange is easy in case of surgically altered anatomy. We considered that EUS-PD is better as the first intervention. For patients in whom passage into the anastomosis site or papilla was not achieved, the distal part of the stent was placed in the MPD and two-thirds of the stent was retained in the stomach side. However, that was effective in preventing stent migration into the pancreatic duct. Thus, a stent length of $15 \mathrm{~cm}$ is considered suitable for EUS-PD.

In the current study, we analyzed short- and long-term outcomes with this new stent for EUS-PD. For short-term outcomes, both the technical and clinical success rates were $100 \%$, similar to that reported in our previous feasibility study [2]. Notably, the rates are obviously higher than those from previous reports $(40 \%-90 \%)[3-8]$. The reasons for the high technical success rate in spite of the small targeted PD (median PD diameter, $3.5 \mathrm{~mm}$, range, $1-14 \mathrm{~mm}$ ) may be attributable to the use of a $22 \mathrm{G}$ needle. A thinner needle allows easier puncture of the small PD, although the following thinner guidewire maneuverability is often difficult because of kinking and bending. The most important concern with EUS-PD is development of AEs. Previous reports have revealed AE rates of $5.6 \%$ to $42.9 \%$ [3-5,14-18]. In most cases, the severity of AEs was mild (e.g., transient postprocedural abdominal pain), but se- vere AEs (e.g., bleeding, pancreatic fistula, and severe pancreatitis) also occurred. In the current study, AEs occurred in $23 \%$ of EUS-PD patients. That rate is relatively lower than the rate in a previous report on EUS-PD or even the rate for surgical treatment (30\%) [19]. Moreover, there was no stent inward migration or pancreatic juice leakage in the current study. That was made possible by the new stent's pigtail anchor together with its four flanges and side holes at the proximal and distal ends but not in the middle part of the stent. However, severe bleeding that required transcatheter arterial embolization was observed in one patient in whom an electrocautery dilator was used for tract dilation. In contrast, there was no bleeding in patients in whom we used an ES dilator, which is an ultraslim mechanical dilator. These results suggest that tract dilation using an electrocautery dilator causes damage to the vessels around the tract by the "burn effect," resulting in unexpected bleeding even with use of the Doppler mode to avoid injuring the intervening blood vessels under EUS guidance.

Apart from EUS-biliary drainage, the reported EUS-PD data are limited because of the limited indications for and difficulty of this procedure. Thus, the long-term follow-up outcome is obscure and the timing of stent removal remains controversial. Ergun et al reported that in $72 \%$ of patients (13/18), symptoms disappeared during the 37 months after the initial procedure, although stent occlusion occurred in $50 \%$ (9/18) [7]. On the other hand, Fujii et al required a median of two times (range, $1-6$ times) of stent exchange in 29 patients during a 23-month follow-up period. When the stent was placed, symptoms completely disappeared in 24 of the 29 patients (82.8\%). Furthermore, 23 patients were finally stent-free during the 32-month follow-up period, although four of them showed symptoms [8]. The current study showed that 20 of 25 patients underwent regular stent exchange a median of three times (range, 1-12 times) in the 23 months after the initial procedure. During the observational period, 10 patients became stent-free (6 pa- 
tients, spontaneous dislodgement; 4 patients, intentional stent removal). All cases of spontaneous stent dislodgement were observed after the EUS-PD stent had been exchanged two or three times. That indicates that after placement of the EUS-PD stent for 6 to 9 months, some patients developed a persistent fistula. Therefore, even after stent dislodgement, five of six patients had no symptom recurrence. In all of these six cases, passing the stricture site was impossible (i. e., pancreaticogastrostomy). In such cases, stent dislodgement may be more likely to occur than in cases involving transenteric antegrade PD stenting. In the current study, we also complied with requests from some patients to have their stent removed a year after it was placed because they had already suffered from ARP for a long time before they were finally referred to our hospital. Notably, 12 patients opted to leave their stents in place and were symptom-free during the observational period. The endpoint for those undergoing prolonged stent exchanges is complete stent removal while achieving symptom relief. However, data are scarce as to when an EUS-PD stent should be removed after symptoms have been relieved. Although we are not sure whether these patients wanted to undergo stent exchange, it appears that it is possible for such individuals to be stent-free after 1 year of stent placement and to have a low recurrence rate. The timing of stent exchange also remains controversial. In some studies, the median time to stent dysfunction is presumed to be 5 to 6 months [4,7]. In the current study, unless spontaneous dislodgement occurred, stent exchange was planned every 3 to 4 months once a stent had been in place for 1 year. However, as shown in $>$ Table 4 , the time interval differed because some patients did not want to have a short-term stent exchange at 3 to 4 months. In the single-center observational study of EUS-PD of 94 patients by Will et al [20], they reported that stents were not routinely changed in patients who had no complaints with transgastric drainage. However, their followup clinical success rate was high. Large-scale studies are needed to further clarify the timing of and necessity for regular stent exchange.

In the current study, disconnected pancreatic duct syndrome was observed in five patients (17\%). Theoretically, the EUS-PD stent must be left in place because of the natural closure of the tract after stent removal, unless recanalization can be achieved in DPDS patients. To date, there have only been a few reports on endotherapy with DPDS. Varadarajulu et al [21] reported long-term outcomes in 97 DPDS patients using endoscopy. The median duration of stent placement was 58 days (range, 4-460 days) and the technical success rate was $55 \%$, with long-term resolution achieved in $94 \%$ of patients. Varadarajulu et al performed regular stent exchange every 6 to 8 weeks to prevent stent occlusion but the timing of it was unclear. Ikenbery et al [22] reported that the stent occlusion rate depended on duration of stent placement, as a stent occlusion rate of $100 \%$ was observed in patients whose stents were removed within 9 weeks. Thus, based on these data and our experience, the optimal timing for transpapillary stent exchange appears to be 3 months or more.

Recently, a self-expandable metal stent dedicated tor EUSPD has been developed [6]. Although it is uncertain whether the newly developed stent is suitable for all patients in whom EUS-PD is needed because of its large-bore 6-mm-diameter, it appears to be traumatic to a nondilated PD as is the less than 3-mm-diameter PD in the current study.

As a limitation, this study was retrospectively conducted on a small number of patients by experts in interventional EUS at a single institution.

\section{Conclusion}

In conclusion, it is feasible to use our recently developed $7 \mathrm{~F}$ plastic stent for EUS-PD and its clinical success over the long term in the majority of the patients has been demonstrated in the current study. A prospective multicenter study is therefore warranted.

\section{Acknowledgements}

The authors are indebted to Associate Professor Edward Barroga, Senior Medical Editor of the Department of International Medical Communications of Tokyo Medical University, for his editorial review of the English manuscript.

\section{Competing interests}

None

\section{References}

[1] Bataille L, Deprez P. A new application for therapeutic EUS: main pancreatic duct drainage with a "pancreatic rendezvous technique". Gastrointest Endosc 2002; 55: 740 - 743

[2] Itoi T, Sofuni A, Tsuchiya T et al. Initial evaluation of a new plastic pancreatic duct stent for endoscopic ultrasonography-guided placement. Endoscopy 2015; 47: 462 - 465

[3] Kahaleh M, Hernandez AJ, Tokar J et al. EUS-guided pancreaticogastrostomy: analysis of its efficacy to drain inaccessible pancreatic ducts. Gastrointest Endosc 2007; 65: 224-230

[4] Tessier G, Bories E, Arvanitakis M et al. EUS-guided pancreatogastrostomy and pancreatobulbostomy for the treatment of pain in patients with pancreatic ductal dilatation inaccessible for transpapillary endoscopic therapy. Gastrointest Endosc 2007; 65: 233-241

[5] Kurihara T, Itoi T, Sofuni A et al. Endoscopic ultrasonography-guided pancreatic duct drainage after failed ERCP in patients with malignant and benign pancreatic duct obstructions. Dig Endosc 2013; 25: (Suppl. 02): 109-116

[6] Oh D, Park DH, Cho MK et al. Feasibility and safety of a fully covered self-expandable metal stent with antimigration properties for EUSguided pancreatic duct drainage: early and midterm outcomes (with video). Gastrointest Endosc 2016; 83: 366-373

[7] Ergun M, Aouattah T, Gillain C et al. Endoscopic ultrasound-guided transluminal drainage of pancreatic duct obstruction: long-term outcome. Endoscopy 2011; 43: 518-525

[8] Fujii LL, Topazian MD, Abu Dayyeh BK et al. EUS-guided pancreatic duct intervention: outcomes of a single tertiary-care referral center experience. Gastrointest Endosc 2013; 78: 854-864 
[9] Cotton PB, Eisen GM, Aabakken L et al. A lexicon for endoscopic adverse events: Report of an ASGE workshop. Gastrointest Endosc 2010; 71: $446-454$

[10] Devière J. EUS-guided pancreatic duct drainage: a rare indication in need of prospective evidence. Gastrointest Endosc 2017; 85: 178 180

[11] Sharma M, Toshniwal J, Vashistha C et al. Shearing of the sheath of the guide wire: A complication of endoscopic ultrasound-guided rendezvous procedure. Endosc Ultrasound 2013; 2: 171-172

[12] François E, Kahaleh M, Giovannini M et al. EUS-guided pancreaticogastrostomy. Gastrointest Endosc 2002; 56: 128-133

[13] Itoi T, Kasuya K, Sofuni A et al. Endoscopic ultrasonography-guided pancreatic duct access: technique and literature review of pancreatography, transmural drainage and rendezvous techniques. Dig Endosc 2013; 25: 241-252

[14] Mallery S, Matlock J, Freeman ML. EUS-guided rendezvous drainage of obstructed biliary and pancreatic ducts: Report of 6 cases. Gastrointest Endosc 2004; 59: 100 - 107

[15] Kikuyama M, Itoi T, Ota Y et al. Therapeutic endoscopy for stenotic pancreatodigestive tract anastomosis after pancreatoduodenectomy (with videos). Gastrointest Endosc 2011; 73: 376-382
[16] Itoi T, Kikuyama M, Ishii K et al. EUS-guided rendezvous with singleballoon enteroscopy for treatment of stenotic pancreaticojejunal anastomosis in post-Whipple patients (with video). Gastrointest Endosc 2011; 73: 398-401

[17] Umeda J, Itoi T. Current status of preoperative biliary drainage. J Gastroenterol 2015; 50: 940 - 954

[18] Tanaka R, Itoi T, Honjyo M et al. New digital cholangiopancreatoscopy for diagnosis and therapy of pancreaticobiliary diseases (with videos). J Hepatobiliary Pancreat Sci 2016; 23: 220-226

[19] Markowitz JS, Rattner DW, Warshaw AL. Failure of symptomatic relief after pancreaticojejunal decompression for chronic pancreatitis. Strategies for salvage. Arch Surg 1994; 129: 374- 379

[20] Will U, Reichel A, Fueldner F et al. Endoscopic ultrasonography-guided drainage for patients with symptomatic obstruction and enlargement of the pancreatic duct. World J Gastroenterol 2015; 21: 13140 13151

[21] Varadarajulu S, Noone TC, Tutuian R et al. Predictors of outcome in pancreatic duct disruption managed by endoscopic transpapillary stent placement. Gastrointest Endosc 2005; 61: 568 - 575

[22] Ikenberry SO, Sherman S, Hawes RH et al. The occlusion rate of pancreatic stents. Gastrointest Endosc 1994; 40: 611-613 